\title{
Investigation of the Internal Rotation in Propargyl Mercaptan by Microwave Spectrum Analysis
}

\author{
F. SCAPPINI* and H. MÄDER \\ Abteilung für Chemische Physik im Institut für Physikalische Chemie der Universität Kiel, \\ Germany \\ and \\ J. SHERIDAN \\ University College of North Wales, Bangor, Great Britain \\ (Z. Naturforsch. 28a, 77-81 [1973]; received 2. December 1972)
}

\begin{abstract}
The microwave investigation of propargyl mercaptan has been continued to study the internal rotation of the $\mathrm{SH}$ group in the molecule. A Hamiltonian is proposed to describe the over-all rotation and the torsion of the top. The analysis leads to the result that the potential function is a double minimum potential with a lower barrier of $470 \pm 10 \mathrm{~cm}^{-1}$ at the cis position and a higher barrier of $2000 \pm 500 \mathrm{~cm}^{-1}$ at the trans position.
\end{abstract}

\section{Introduction}

The microwave spectrum of propargyl mercaptan, Fig. 1, was shown by Bolton and Sheridan ${ }^{1}$ to correspond to the gauche configuration, Fig. 2. A striking series of $\mu_{\mathrm{c}}$-lines were analysed as rotation-vibration transitions between the two lowest states of the $\mathrm{SH}$-torsion mode, and the separartion of these two vibrational levels was shown, with accuracy, to be only some $6890 \mathrm{MHz}$. In consequence, the $\mu_{\mathrm{a}}$ - and $\mu_{\mathrm{h}}$-transitions appeared as close doublets of equal intensity, and, although, in the tabulation of the data, assignment of certain members of these doublets to one or other of the two vibrational states could be tentatively made, and sometimes deduced with certainty, a detailed analysis of the doublets was deferred, pending an accurate treatment of the perturbations between the energy level manifolds of the two states, the effects of which were obvious for certain transitions. BolTON and BARON (private communication, to be separately published) have refined considerably the treatment of the perturbations, and have been kind enough to provide us with details of these computations.

Reprint requests to Dr. H. MäDER,, Institut für Physikalische Chemie der Universität Kiel, Abt. Chem. Physik, D-2300 Kiel, Olshausenstraße 40-60.

* On leave from Laboratorio di Spettroscopia Molecolare, Bologna, Italy.

1 K. Bolton and J. Sheridan, Spectrochim. Acta 26A, 1001 [1970].
In the present work, we report the first measurements of the lowest rotational transitions, from which a very accurate value of $\Delta$, the energy between the ground level and the first torsional leve, is derived, and we have made a preliminary determination of dipole moment components from Stark effects. The spectrum of the deuterated species, $\mathrm{HCCCH}_{9} \mathrm{SD}$, is also assigned, allowing a closer estimate of the equilibrium dihedral angle of the $\mathrm{SH}$-group, and we propose a potential function for the $\mathrm{SH}$-torsion on the basis of refined measurements of a number of transitions.

\section{Experimental}

The spectra have been recorded in the region from 5 to $40 \mathrm{GHz}$ with a Stark modulation microwave spektrograph, employing phase stabilized BWO's as radiation sources ${ }^{2}, 3$. The radio frequency microwave double resonance (RFMDR) technique was applied in the way described elsewhere 4, 5, 6 .

First we have analyzed the spectrum of the deuterated species, $\mathrm{HCCCH}_{2} \mathrm{SD}$ (it will be called the "Dspecies"). The deuteration was carried out directly in

2 H. D. Rudolph, Z. Angew. Phys. 13, 401 [1961].

3 U. Andresen and H. Dreizler, Z. Angew. Phys. 30, 207 [1970].

4 F. J. WodarczyK and E. B. Wilson, J. Mol. Spectrosc. 37, 445 [1971].

5 F. SCAppini and A. Guarnieri, Z. Naturforsch. 27a, 1011 [1972].

- R. Schwarz, unpublished results. 
the absorption cell at a temperature of about $-60^{\circ} \mathrm{C}$. By using the RFMDR technique the $\mu_{\mathrm{a}}$-spectrum was assigned and the $B$ and $C$ rotational constants evaluated, Tab. 1. The $\mu_{\mathrm{h}}$-spectrum was not found, probably because of the weakness of the lines due to the small dipole moment component and to the incomplete deuteration. The measured lines did not show any internal rotation splitting, indicating that the separation between the ground and the first torsional level is less than in the case of the $\mathrm{H}$-species.

Table 1. Observed and calculated $\mu_{\mathrm{a}}$-transitions of $\mathrm{HCCCH}_{2} \mathrm{SD}$ in $\mathrm{MHz}$. Rotational constants in $\mathrm{MHz}$. The frequencies were calculated using a rigid rotor procedure.

\begin{tabular}{ccl}
\hline Transition & $\begin{array}{l}\text { Observed } \\
\text { frequency }\end{array}$ & $\begin{array}{l}\text { Calculated } \\
\text { frequency }\end{array}$ \\
\hline $3_{12} \leftarrow 2_{11}$ & 17610.664 & 17610.664 \\
$4_{22} \leftarrow 3_{21}$ & 22953.946 & 22955.127 \\
$4_{04} \leftarrow 3_{03}$ & 22888.695 & 22889.383 \\
$4_{31} \leftarrow 3_{30}$ & 22931.417 & 22930.512 \\
$4_{32} \leftarrow 3_{31}$ & 22931.417 & 22930.355 \\
$4_{23} \leftarrow 3_{22}$ & 22920.826 & 22920.923 \\
\hline$A^{*}=--; B=3005.5 \pm 0.2 ; C=2725.4 \pm 0.2$.
\end{tabular}

* The value of the $A$ rotational constant obtained from the strucure fitting was $\mathrm{A}=20349 \mathrm{MHz}$.

The measurement of the $\mathrm{O}_{00} \rightarrow 1_{01}$ transition for the $\mathrm{H}$-species, which was missing in the previous work because of its low frequency, allowed us to calculate exactly the splitting for the $\mathrm{H}$-species between the ground and the first torsional level: $\Delta=6891.76 \pm$ $0.02 \mathrm{MHz}$.

In this work some additional transitions of the $\mathrm{H}$ species have been measured making use of the RFMDR whenever possible. Furthermore other transitions were remeasured in order to resolve the doublets, which had not been previously resolved, or possibly to improve the frequency measurements, Table 3.

From the Stark effect of some low J lines and using a simple rigid rotor treatment, we obtained preliminary results for the in-plane dipole moment components of the H-species: $\mu_{\mathrm{a}}=0.74 \pm 0.02 \mathrm{D}, \mu_{\mathrm{h}}=$ $0.58 \pm 0.01 \mathrm{D}$. These values are averaged between the ground and the first torsional level and the errors are defined within the procedure used. A thorough treatment taking into account both the rotation and the torsion is in progress.

With the rotational constants of the $\mathrm{H}$ - and Dspecies, assuming the acetylene structure ${ }^{7}$, a structure fitting was made and the internal rotation angle corresponding to the gauche position was evaluated to be: $\alpha_{\min }=127^{\circ} \pm 1^{\circ}$ (measured from the trans position). The $A$ rotational constant given in Table 1 results also from this structure.

7 "Tables of Interatomic Distances and Configuration in Molecules and Ions". The Chemical Society, London 1965.

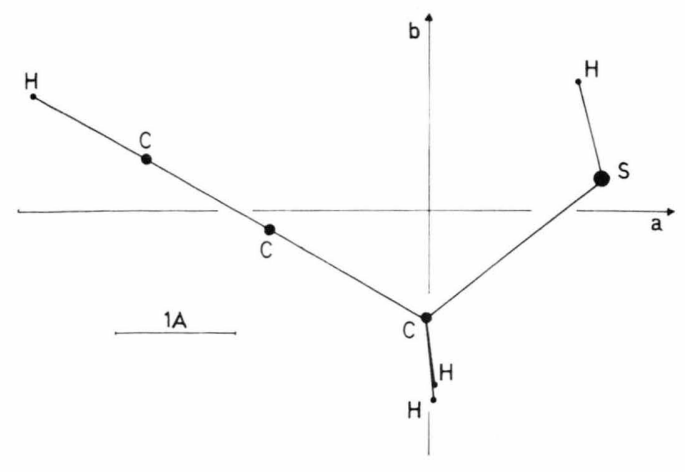

Fig. 1. Propargyl mercaptan in the a-b plane.

The spectrum of the trans rotamer, Fig. 2, was also searched for at different temperatures, but no evidence of this form was found.

\section{Hamiltonian for the Rotation and the Internal Rotation}

Following the same approximation that Hirota used in the case of propargyl alcohol ${ }^{8}$, we have treated the $\mathrm{SH}$ group as a symmetric top rotating about the C-S bond. The justification for this approximation is that the framework is heavy compared to the top.

The Hamiltonian that we have used is the following:

with

$$
\mathcal{H}=H_{\mathrm{R}}+H_{\mathrm{T}}+H_{\mathrm{RT}}
$$

$$
\begin{aligned}
& H_{\mathrm{R}}=A^{0} P_{\mathrm{o}}{ }^{2}+B^{0} P_{\mathrm{b}}{ }^{2}+C^{0} P_{\mathrm{c}}{ }^{2}+F\left(\varrho_{\mathrm{o}}{ }^{2} P_{\mathrm{o}}{ }^{2}+\varrho_{\mathrm{b}}{ }^{2} P_{\mathrm{b}}{ }^{2}\right) \\
& +F \varrho_{\mathrm{a}} \varrho_{\mathrm{b}}\left(P_{\mathrm{a}} P_{\mathrm{b}}+P_{\mathrm{b}} P_{\mathrm{a}}\right), \\
& H_{\mathrm{T}}=F_{\mathrm{p}_{\alpha}}{ }^{2}+\frac{1}{2} V_{1}(1-\cos \alpha)+\frac{1}{2} V_{2}(1-\cos 2 \alpha) \\
& H_{\mathrm{RT}}=-2 F \mathrm{p}_{\alpha}\left(\varrho_{\mathrm{a}} P_{\mathrm{a}}+\varrho_{\mathrm{b}} P_{\mathrm{b}}\right) \\
& +\frac{1}{2} V_{3}(1-\cos 3 \alpha)
\end{aligned}
$$

in which $H_{\mathrm{R}}$ is the rotational part, $H_{\mathrm{T}}$ the torsional part, $H_{\mathrm{RT}}$ the rotation-torsion interaction part; $A^{0}$, etc. are rotational constants; $\varrho_{\mathrm{a}}=\lambda_{\mathrm{a}} I_{\alpha} / I_{\mathrm{a}}$, etc.; $\lambda_{\mathrm{a}}$, etc. are the direction cosines between the internal rotation axis and the principal axes of the entire molecule; $I_{\alpha}$ is the moment inertia of the top about the internal

${ }^{8}$ E. Hirota, J. Mol. Spectrosc. 26, 335 [1968]. 


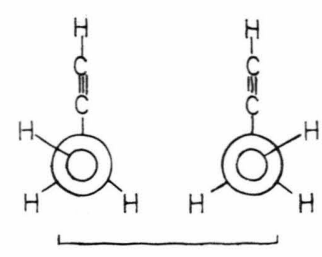

GAUCHE

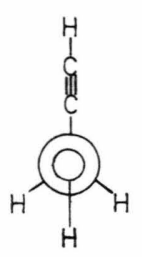

TRANS
Fig. 2. Gauche and trans rotamers of propargyl mercaptan.

rotation axis; $F$ is the inverse reduced moment of inertia of the top; and $p_{\alpha}=-i \partial / \partial \alpha$.

At this point, by simple consideration based on group theory, it is possible to explain the selection rules governing the pure rotation transitions and the torsion-rotation transitions of this molecule. The $\mathrm{Ha}$ miltonian (1) belongs to the invariance group $C_{\mathrm{i}}$, which is a subgroup of the direct product $C_{s} \otimes V$. The group $C_{i}$ has the following character table:

\begin{tabular}{rrrr}
\hline$C_{i}$ & E & i \\
\hline A $_{+}$ & 1 & 1 \\
A. & 1 & -1 \\
\hline
\end{tabular}

with the generating elements ${ }^{9}$ ( $I^{\mathrm{r}}$ representation)

$$
\begin{array}{ll}
\text { E: } & \alpha \rightrightarrows \alpha, \varphi \rightrightarrows \varphi, \vartheta \rightrightarrows \vartheta, \chi \rightarrow \chi, p_{\alpha} \rightarrow p_{\alpha} \\
& P_{\mathrm{a}} \rightrightarrows P_{\mathrm{a}}, P_{\mathrm{b}} \rightarrow P_{\mathrm{b}}, P_{\mathrm{c}} \rightarrow P_{\mathrm{c}} \\
\mathrm{i}=\sigma_{\mathrm{h}} \mathrm{C}_{\mathbf{2}}: & \alpha \rightarrow-\alpha, \varphi \rightarrow \varphi+\pi, \vartheta \rightarrow \pi-\vartheta, \chi \rightarrow-\chi \\
& P_{\alpha} \rightarrow-p_{\alpha}, P_{\mathrm{a}} \rightarrow-P_{\mathrm{a}}, P_{\mathrm{b}} \rightarrow-P_{\mathrm{b}}, P_{\mathrm{c}} \rightarrow P_{\mathrm{c}}
\end{array}
$$

The dipole moment components all belong to the A symmetry species. The eigenfunctions of the $\mathrm{Ha}$ miltonian (1) must belong either to $A_{+}$or $A_{-}$. They are linear combinations of the product of the torsional functions, $U_{\mathrm{v} \pm}(\alpha)$, and the asymmetric rotor functions $\psi_{J K}{ }_{-1} K_{+1} M$. The torsional functions $U_{\mathrm{v} \pm}(\alpha)$ can be easily specified under the group $C_{\mathrm{i}}$, the + and - signs characterizieg the symmetry of the torsional levels $v$ according to $\mathrm{A}_{+}$and $\mathrm{A}_{-}$, respectively. The rotational

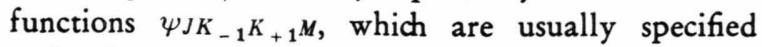
under the group $V$, can be correlated to the species of the group $C_{i}$ by the following correlation table:

\begin{tabular}{ccc}
\hline$C_{\mathrm{i}}$ & $V$ & $K_{{ }_{1}} K_{+1}$ \\
\hline $\mathrm{A}_{+}$ & $\mathrm{A}$ & ee \\
& $\mathrm{B}_{\mathrm{c}}$ & oe \\
& $\mathrm{B}_{\mathrm{b}}$ & oo \\
$\mathrm{A}-$ & $\mathrm{B}_{\mathrm{a}}$ & eo \\
\hline
\end{tabular}

Figure 3 indicates the species of some ro-torsional levels of $\mathrm{HCCCH}_{2} \mathrm{SH}$ as they follow from the above considerations $\left(\mathrm{A}_{+}\right.$and $\mathrm{A}_{-}$being replaced by + and -, respectively, as it will be done later on).
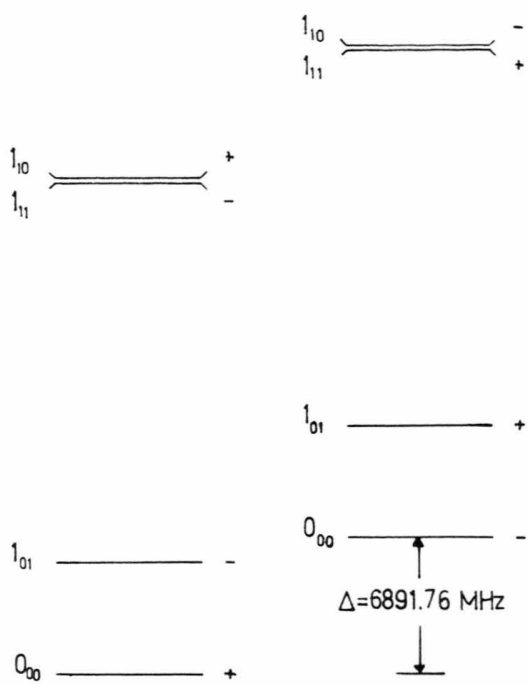

$0_{\infty}$<smiles>[13IH]</smiles><smiles>[OH2+]</smiles><smiles>[O-]</smiles>

Fig. 3. Energy level pattern for the ground state $\left(0^{+}\right)$and for the first torsional state (0-) of $\mathrm{HCCCH}_{2} \mathrm{SH}$. The symmetry of the ro-torsional levels according to the species of group $C_{i}$ is shown by + and - corresponding to $A_{+}$and $A_{-}$.

For the transition moment to be non-vanishing the selection rules are:

$$
-\longleftrightarrow+\quad ; \quad \pm \leftarrow \rightarrow \pm
$$

Now it is straightforward to see that the $\mu_{\mathrm{a}}$ and $\mu_{\mathrm{b}}$ transitions can only connect ro-torsional levels of the same torsional species and, therefore, in the microwave region, they are pure rotation transitions. On 
the other hand the $\mu_{\mathrm{c}}$ transitions can only connect rotorsional levels of different torsional species and, therefore, they are torsion-rotation transitions.

\section{Solution of the Hamiltonian}

The sequence of our calculations was the following. First the matrix corresponding to the Hamiltonian $\mathrm{H}_{\mathrm{T}}$ was set up in the free rotor functions: $(2 \pi)^{-\frac{1}{2}} \exp$ (ima). The diagonalization of this matrix yielded the torsional eigenfunctions $U_{\mathrm{v} \pm}(\alpha)$. The matrix corresponding to the total Hamiltonian (1) was then set up in the product basis: $U_{\mathrm{v}_{ \pm}} \cdot \psi \times_{J K M}$, where the symmetric rotor functions, $\psi x_{J K M}$, were chosen for simplicity instead of the asymmetric rotor functions, $\psi_{J K}{ }_{-1} K_{+1} M$. This matrix was diagonalized using that set of rotational constants, $A^{0}, B^{0}, C^{0}$, which best reproduced the absolute frequencies of the lines listed in Table 3. The heights of the cis and trans barrier, $V_{\text {cis }}$ and $V_{\text {trans }}$, were calculated by least squares fitting the experimental splitting $\Delta$ and the splittings of the lines, Table 3.

At this point we realized that a better fitting of the line splitting could be obtained using two slightly dif- ferent sets of rotational constants, $A^{0}+\Delta A^{0}$, etc., and $A^{0}-\triangle A^{0}$, etc. for the + and - torsional levels, respectively. This treatment can be thought of as a first order approximation to the dependence of the rotational constants on the torsion.

A final least squares fit was made using the increments of the rotational constants, $\Delta A^{0}, \Delta B^{0}, \Delta C^{0}$, as fitting parameters.

Table 2 gives all the constants that enter into the Hamiltonian (1) and the heights of the cis and trans

Table 2. Values of the constants which enter into the Hamiltonian (1). $A^{\circ}, B^{\circ}, C^{\circ}, \Delta A^{\circ}, \Delta B^{\circ}, \Delta C^{\circ}$ are in $\mathrm{MHz}$; $F, V_{1}, V_{2}, V_{3}$ are in $\mathrm{cm}^{-1} ; \alpha_{\min }$ is in degrees. Values of $V_{\text {cis }}$ and $V_{\text {trans }}$ are in $\mathrm{cm}^{-1}$.

\begin{tabular}{lll}
\hline$A^{\circ}$ & $=$ & 22346.70 \\
$B^{\circ}$ & $=$ & 3046.50 \\
$C^{\circ}$ & $=$ & 2756.50 \\
$\Delta A^{\circ}$ & $=$ & 0.722 \\
$\Delta B^{\circ}$ & $=$ & -0.108 \\
$\Delta B^{\circ}$ & $=$ & -0.026 \\
$F$ & $=$ & 9.115 \\
$\alpha_{\text {min }}$ & $=$ & $127^{\circ} \pm 1^{\circ}$ \\
$V_{1}$ & $=$ & -2071 \\
$V_{2}$ & $=$ & -564 \\
$V_{3}$ & $=$ & 540 \\
$V_{\text {cis }}$ & $=$ & $470 \pm 10$ \\
$V_{\text {trans }}$ & $=$ & $2000 \pm 500$ \\
\hline
\end{tabular}

Table 3. Observed and calculated frequencies in $\mathrm{MHz}$ for the $\mathrm{O}^{+}$and $\mathrm{O}^{-}$torsional states of $\mathrm{HCCCH}_{2} \mathrm{SH}$. Observed and calculated splittings in $\mathrm{MHz}$. The calculations are based on the Hamiltonian (1) and on the constants given in Tab. 2. For the calculated frequencies the centrifugal distortion was not taken into account.

\begin{tabular}{|c|c|c|c|c|c|c|}
\hline \multirow[b]{2}{*}{ Transition } & \multicolumn{2}{|c|}{$0^{-}$} & \multicolumn{2}{|c|}{$0^{+}$} & \multirow{2}{*}{$\begin{array}{l}\text { Obs. Splitting } \\
\left(v_{0^{+}}-v_{0^{-}}\right)\end{array}$} & \multirow{2}{*}{$\begin{array}{l}\text { Calc. Splitting } \\
\left(v_{0}+-v_{0}-\right)\end{array}$} \\
\hline & $\begin{array}{l}\text { Observed } \\
\text { frequency }\end{array}$ & $\begin{array}{l}\text { Calculated } \\
\text { frequency }\end{array}$ & $\begin{array}{l}\text { Observed } \\
\text { frequency }\end{array}$ & $\begin{array}{l}\text { Calculated } \\
\text { frequency }\end{array}$ & & \\
\hline $\begin{array}{l}1_{01} \leftarrow 0_{00} \\
1_{11} \leftarrow 0_{00} \\
1_{10} \leftarrow 1_{01} \\
2_{11} \leftarrow 1_{10} \\
2_{12} \leftarrow 1_{11} \\
2_{02} \leftarrow 1_{01} \\
2_{12} \leftarrow 1_{01} \\
2_{11} \leftarrow 2_{02} \\
3_{21} \leftarrow 2_{20} \\
3_{22} \leftarrow 2_{21} \\
3_{12} \leftarrow 2_{11} \\
3_{03} \leftarrow 2_{02} \\
3_{13} \leftarrow 2_{02} \\
3_{12} \leftarrow 3_{03} \\
4_{22} \leftarrow 3_{21} \\
4_{23} \leftarrow 3_{22} \\
4_{04} \leftarrow 3_{03} \\
4_{13} \leftarrow 4_{04} \\
5_{05} \leftarrow 4_{04} \\
6_{33} \leftarrow 5_{32} \\
6_{34} \leftarrow 5_{33} \\
6_{06} \leftarrow 5_{05}\end{array}$ & $\begin{array}{rl}5 & 803.180 \\
25 & 104.853 \\
19 & 589.860 \\
11 & 894.976 \\
11 & 317.920 \\
11 & 603.184 \\
30 & 619.893 \\
19 & 881.740 \\
17 & 423.489 \\
17 & 410.731 \\
17 & 838.834 \\
17 & 396.655 \\
35 & 992.663 \\
20 & 324.019 \\
23 & 243.592 \\
23 & 211.636 \\
23 & 180.376 \\
20 & 920.314 \\
28 & 951.208 \\
34 & 841.238 \\
34 & 838.754 \\
34 & 705.942\end{array}$ & $\begin{array}{rl}5 & 803.134 \\
25 & 105.010 \\
19 & 589.970 \\
11 & 894.226 \\
11 & 318.146 \\
11 & 603.031 \\
30 & 620.023 \\
19 & 881.166 \\
17 & 422.466 \\
17 & 409.881 \\
17 & 838.196 \\
17 & 396.456 \\
35 & 991.737 \\
20 & 322.905 \\
23 & 242.332 \\
23 & 210.888 \\
23 & 180.189 \\
20 & 919.802 \\
28 & 951.048 \\
34 & 857.794 \\
34 & 836.611 \\
34 & 705.943\end{array}$ & $\begin{array}{rr}5 & 802.829 \\
25 & 103.544 \\
19 & 588.750 \\
11 & 893.944 \\
11 & 318.220 \\
11 & 602.272 \\
30 & 618.498 \\
19 & 880.540 \\
17 & 422.251 \\
17 & 409.489 \\
17 & 840.139 \\
17 & 395.395 \\
35 & 995.309 \\
20 & 325.100 \\
23 & 241.864 \\
23 & 210.040 \\
23 & 178.780 \\
20 & 927.538 \\
28 & 949.236 \\
34 & 840.133 \\
34 & 837.667 \\
34 & 703.737\end{array}$ & $\begin{array}{rl}5 & 802.713 \\
25 & 103.679 \\
19 & 588.521 \\
11 & 893.658 \\
11 & 318.645 \\
11 & 602.201 \\
30 & 619.611 \\
19 & 879.978 \\
17 & 421.377 \\
17 & 408.791 \\
17 & 838.899 \\
17 & 395.239 \\
35 & 994.435 \\
20 & 323.638 \\
23 & 178.613 \\
23 & 240.848 \\
23 & 209.421 \\
20 & 926.998 \\
28 & 949.146 \\
34 & 834.473 \\
34 & 703.747 \\
34 & 835.563\end{array}$ & $\begin{array}{r}0.351 \\
1.309 \\
1.110 \\
1.032 \\
-0.300 \\
0.912 \\
1.395 \\
1.200 \\
1.238 \\
1.242 \\
-1.305 \\
1.260 \\
-2.646 \\
-1.081 \\
1.728 \\
1.596 \\
1.596 \\
-7.224 \\
1.972 \\
1.105 \\
1.087 \\
2.205\end{array}$ & $\begin{array}{r}0.421 \\
1.331 \\
1.449 \\
0.568 \\
-0.499 \\
0.830 \\
0.412 \\
1.188 \\
1.089 \\
1.090 \\
-0.703 \\
1.217 \\
-2.698 \\
-0.733 \\
1.484 \\
1.467 \\
1.576 \\
-7.196 \\
1.902 \\
2.231 \\
2.138 \\
2.196\end{array}$ \\
\hline
\end{tabular}


barriers. Table 3 gives the observed and calculated values of the frequencies and of the splittings of the $\mu_{\mathrm{a}}$ and $\mu_{\mathrm{b}}$-lines for the $\mathrm{H}$-species. Table 4 gives the rotational constants of the $\mathrm{H}$-species corresponding to the ground $\left(\mathrm{O}^{+}\right)$and to the first torsional level $\left(\mathrm{O}^{-}\right)$, as obtained from the low $J$ transitions.

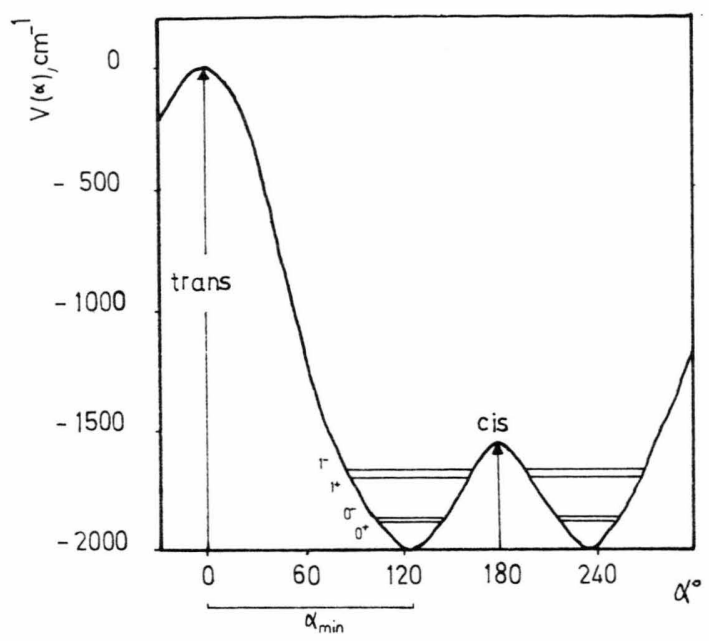

Fig. 4. Potential function for the internal rotation in propargyl mercaptan. The torsional levels $\mathrm{O}^{+}, \mathrm{O}^{-}, 1^{+}, 1^{-}$for the $\mathrm{H}$-species are indicated.

\section{Discussion}

The potential function, as shown in Fig. 4, is a double minimum potential and the internal rotation splitting $\Delta$ is due to tunnelling of the two gauche
Table 4. Rotational constants in $\mathrm{MHz}$ and asymmetry parameter for the $\mathrm{O}^{+}$and $\mathrm{O}^{-}$states of $\mathrm{HCCCH}_{2} \mathrm{SH}$, by using a rigid rotor fitting procedure.

\begin{tabular}{ccc}
\hline & $C^{+}$ & $0^{-}$ \\
\hline$A$ & $22347.56 \pm 0.05$ & $22346.39 \pm 0.05$ \\
$B$ & $3045.80 \pm 0.03$ & $3045.47 \pm 0.03$ \\
$C$ & $2757.39 \pm 0.03$ & $2757.37 \pm 0.03$ \\
\hdashline & -0.97055 & -0.97059 \\
\hline
\end{tabular}

forms through the cis barrier. This explains why the splitting $\Delta$ and consequently the splittings of the lines are much more sensitive to the cis barrier than to the trans barrier, and why the trans barrier remains rather undetermined.

The results shown in Tab. 3 indicate a reasonable agreement between the experimental splittings of the lines and those calculated using the Hamiltonian (1). This allows us to say with confidence that the simplified Hamiltonian (1) is a good one for studying the internal rotation in propargyl mercaptan. It could as well be used for studying other similar molecules.

The authors wish to thank Prof. H. Dreizler and Prof. A. Guarnieri for many helpful discussions, MSc. L. Charpentier for assistance in preparing the manuscript and the Rechenzentrum der Universität Kiel for free computer time. Financial support of the Deutsche Forschungsgemeinschaft and the Fonds der Chemie is also acknowledged.

F. ScAppini thanks the British Science Research Council for a Research Fellowship, during the tenure of which this work was begun. 